\title{
МОДЕЛИРОВАНИЕ ПРОЦЕССОВ ИНФОРМАЦИОННО-ПСИХОЛОГИЧЕСКОГО ВОЗДЕЙСТВИЯ В СОЦИАЛЬНЫХ СЕТЯХ
}

\author{
И. В. Гончаров, П. А. Паринов, А. А. Сирота \\ Воронежский государственный университет
}

Поступила в редакцию 01.05.2018 г.

\begin{abstract}
Аннотация. Рассмотрены модели социальных сетей, а также следующие классы задач, связанные с исследованием информационно-психологического воздействия: моделирование информационного влияния, информационного управления и информационного противоборства. Предложены модель распространения информационно-психологического воздействия в социальных сетях на основе клеточных автоматов, иерархическая структура изменения состояний субъекта информационно-психологического воздействия и схема переходов из состояния в состояние, положенные в основу алгоритма работы клеточного автомата. Предложенный клеточный автомат позволяет учитывать социально-психологические факторы субъектов социальной сети и моделировать распространение информационно-психологического воздействия в социальной сети. Проведено моделирования предложенного алгоритма. Приведены результаты моделирования предложенного алгоритма.

Ключевые слова: социальная сеть, информационно-психологическое воздействие, негативное ИПВ, позитивное ИПВ, клеточные автоматы, распространение, социально-психологические факторы.

Annotation. The models of social networks are considered, as well as the following classes of problems related to the study of information and psychological impact: modeling of information influence, information management and information confrontation. A model for the spread of information and psychological effects in social networks based on cellular automata is proposed, the hierarchical structure of the changes in the states of the subject of information-psychological influence, and the scheme of transitions from the state to the state that form the basis for the algorithm for the operation of the cellular automaton. The proposed cellular automaton allows to take into account the socio-psychological factors of social network subjects and to simulate the spread of information and psychological impact in the social network. The proposed algorithm is simulated. The results of modeling the proposed algorithm are presented.

Keywords: social network, information-psychological impact, negative IPI, positive IPI, cellular automata, distribution IPI, socio-psychological factors.
\end{abstract}

\section{ВВЕДЕНИЕ}

Информационно-психологическое воздействие может быть определено как информационное воздействие на психику человека, оказывающее влияние на восприятие им реальной действительности, в том числе на его поведенческие функции, а также, в некоторых случаях, на функционирование органов и систем человеческого организма $[1,2,3]$. Информационно-психологическое воздействие (ИПВ) может оказывать влияние, как на ин-

(ㄱ Гончаров И. В., Паринов П. А., Сирота А. А., 2018 дивидуальное, так и на групповое, массовое и общественное сознание. При этом ИПВ может носить как позитивный, так и негативный характер, в зависимости от преследуемых целей. ИПВ позитивного характера может осуществляться для лечения и реабилитации людей, мотивации к изменению норм поведения в лучшую сторону, для решения созидательных задач и объединения людей в благородных целях. Негативное ИПВ может применяться для явного или скрытого побуждения человека, групп или общества к действиям в ущерб к их собственным интересам или интересам других. Негативное ИПВ мо- 
жет вызывать психоэмоциональную и социально-психологическую напряженность в обществе, искажение нравственных критериев и норм поведения, морально-политическую дезориентацию, и как следствие приводить к глубокой трансформации индивидуального, группового, массового и общественного сознания, изменения морально-политического и социально-психологического климата в обществе $[1,2,3]$.

Информационно-психологические воздействия могут осуществляться с помощью различных методов и средств. В настоящее время большую активность имеют негативные информационно-психологические воздействия, оказывающие влияние на человека, группу или общество посредством информационно-телекоммуникационных сетей, средств массовой информации, социальных сетей. Данные ИПВ осуществляются с целью: управления обществом, навязывания ему определенного мнения к какой-либо проблеме или ситуации; вербовки новых членов в секты, террористические организации; изменения психического состояния человека. Примером тому служат «цветные революции» в ряде стран, «группы смерти», активная пропагандистская кампания для вербовки иностранных боевиков-террористов с использованием сюжетов из кинофильмов и видеоигр, ориентированных, в основном на людей молодого возраста.

В связи с этим актуальной задачей является моделирование ИПВ в социальных сетях с целью дальнейшего анализа и выработки эффективных методов и средств использования ИПВ в случае позитивного влияния или противодействия им в случае негативного влияния $[1,2,4]$.

\section{1. АНАЛИЗ РЕЗУЛЬТАТОВ ПРЕДШЕСТВУЮЩИХ РАБОТ}

Социальную сеть в известных источниках обычно представляют как граф с множеством вершин (агентов) и ребер, отражающих связи между ними. В качестве агентов могут выступать различные субъекты, как индивидуумы, так и коллективы (группы, организации, сообщества). Под связью понимаются отношения между агентами, например, обмен информацией, социальные отношения, коммуникация $[4,5,6,7,8,9]$. Процесс ИПВ можно разделить на две части: непосредственно распространение ИПВ и изменение мнений агентов сети. При рассмотрении моделей социальных сетей в работе [4] выделяют следующие классы задач, связанных с исследованием ИПВ: моделирование информационного влияния, информационного управления и информационного противоборства.

Модель информационного влияния позволяет исследовать поведение субъекта, на которого производится информационное воздействие. Влияние может быть целенаправленным и нецеленаправленным. Социальное влияние проявляется в процессе коммуникации и сравнения. На основе модели информационного влияния, можно решать задачу информационного управления, т.е. каким должно быть информационное воздействие с точки зрения управляющего субъекта, чтобы добиться требуемого поведения от управляемого субъекта. Используя задачу информационного управления, можно моделировать информационное противоборство, т.е. взаимодействие нескольких субъектов, обладающих несовпадающими интересами и осуществляющих информационные воздействия на один и тот же управляемый субъект [4]. Известны следующие подходы к моделированию влияния.

1. Модели с порогами и модели независимых каскадов $[4,8,10,11,12,13]$. В данном классе моделей субъект - узел сети (вершина графа) может находиться в активном состоянии и неактивном состоянии. Возможен переход из неактивного состояния в активное, но обратный переход не допускается. Агент становится активным в зависимости от выбранного порога. Порог перехода может быть как фиксированным для всех агентов, так и выбираться случайно согласно некоторому вероятностному распределению. Данные модели не учитывают возможность наличия групп, игрового взаимодействия субъектов, индивидуальную активность субъектов, неполную информированность субъектов. 
2. Модели сетевой автокорреляции. В этих моделях мнение и поведение субъекта изменяются под влиянием мнения окружающих его субъектов и являются ответной реакцией субъекта на ИПВ $[14,15,16,17,18,19]$. В указанных работах рассматривается детерминированный дискретно-временной линейный процесс, в котором мнения (свойства) субъекта представлены вектором $y_{t}$ и изменяются под влиянием других субъектов, на основе так называемой матрицы влияния $W: y_{t+1}=W y_{t}$.

3. Модели Изинга $[20,21]$. Введенная изначально для понимания природы ферромагнетизма модель Изинга служит полигоном для проверки методов численного моделирования различных явлений. Для рассмотрения распространения ИПВ в социальных сетях данная модель служит как средство описания изменения поведения в большой социальной группе под влиянием ближайших соседей. В данном случае влияние ближайших соседей является определяющим, а аналогом температуры является готовность группы субъектов социальной сети принять новую идею.

4. Модели влияния на основе цепей Маркова. Эти модели, используя соответствующий математический аппарат, моделируют действия каждого субъекта и группы в целом и позволяют анализировать социальную динамику для выявления шаблонов возникающего группового поведения. В работах [22, $23,24]$ рассматривались условия сходимости мнений субъектов, скорость сходимости мнений $[7,24]$, условия единственного итогового мнения в работах $[7,25]$.

Следует отметить, что все рассмотренные классы моделей отражают правила взаимодействия между субъектами или их группами. Сетевой характер влияния, его свойства и специфику процессов взаимодействия данные модели практически не отражают или отражают не полно.

При рассмотрении социальной сети как множества агентов $[4,26,27,28]$ каждый агент обладает определенным влиянием на других агентов, поэтому возникает потребность в выявлении небольшого числа агентов, обладающих наибольшим влиянием - проблема максимизации влияния $[4,10,29]$. Данные агенты могут использоваться: в качестве точек влияния на других субъектов социальной сети; для мониторинга социальной сети, с целью определения наличия ИПВ. При этом проблема определения самых влиятельных агентов рассматривалась в различных работах при решении следующих задач:

- вирусного маркетинга [29], где социальная сеть представляется сетью Маркова и каждый агент А имеет свою ценность, зависящую от прибыли от продаж другим агентам, на которых окажет влияние агент A;

- максимизации влияния [10] в моделях распространения нововведений, в которых имеется множество активных агентов, причем в некоторый момент времени новый активный агент получает шанс активировать своих соседний с заданной вероятностью;

- моделирования процесса голосования [9], где на каждом шаге каждый агент может изменить свое мнение, случайно выбрав одного из соседей, приняв его мнение. Агент с большей вероятностью изменит свое мнение на мнение, поддерживаемое большинством его соседей.

Помимо задач анализа влияния, управления и противоборства, выделяют задачи распространения информационно-психологического воздействия в информационном пространстве [5]. Информация может распространяться в общем случае [5, 30]: от субъекта к субъекту; от субъекта группе; от центров формирования информации к отдельному субъекту или группе субъектов.

В работах $[5,26,27,28,31]$ авторами предложена мультиагентная модель распространения информации. В данной модели учтена эволюция популяции агентов в течение времени. Отдельные субъекты могут самозарождаться, порождать новых агентов, исчезать из пространства субъектов, получать ссылки от других агентов.

В работах $[5,30]$ жизненный цикл информационных потоков описывается моделью диффузии информации, построенной с помощью методов клеточных автоматов. В данных моделях каждая клетка клеточного автомата может иметь различные состояния, например, «воздействие принято», «воздействие не 
актуально», «воздействие отклонено». Распространение информации происходит на основе вероятностных правил. Рассматриваемые состояния объекта синхронно изменяются через дискретные интервалы времени в соответствии с неизменными локальными вероятностными правилами, которые могут зависеть от состояния переменных, описывающих ближайших соседних субъектов в окрестности данного агента, а также возможно, от состояния самого субъекта. Так, например, в $[8,32]$ моделируется эффект передачи информации «из уст уста» с учетом сильных и слабых связей субъектов.

Также для изучения распространения информации в работах $[6,33,34]$ распространение информации сравнивают с распространением вирусов и применяются модели просачивания и заражения, например SIR модели, SIRS модели и другие.

В работе [35] проводится аналогия между структурой социальных сетей с нейронными сетями. В качестве нейронов рассматриваются отдельные пользователи. На основе данных об активности пользователей осуществляется прогноз новостей, которые могут их заинтересовать. В [35] также предлагается использовать нейронные сети с целью прогнозирования поведения субъекта ИПВ, прогнозирования податливости вербовке субъекта для выполнения каких-либо задач, определение благонадежности субъекта, на основе данных из социальных сетей.

В сфере информационной безопасности необходимо как можно раньше обнаружить информационно-психологическое воздействие. Для этого в работах $[4,37]$ предлагается отслеживать состояния небольшой части узлов сети на основе графовых моделей, проблема состоит в определении множества контролируемых узлов. Нарушение типовой динамики распространения некоторых информационных сообщений может свидетельствовать о наличии информационно-психологического воздействия. Для анализа динамики распространения информации, с целью выявления потоков, вызванных влиянием внешних факторов, могут быть использованы методы вейвлет-анализа $[5,15,17]$.
В работе [5] вводится понятие информационной резервации - изолированной части информационного пространства, и предложены модификации модели диффузии информации для моделирования динамики информационных потоков в информационных резервациях. Информационные резервации представляют собой информационное пространство, находящееся под непрерывным информационно-психологическим воздействием, и могут применяться для информационно-психологического управления социумом.

В тоже время, следует отметить, рассмотренные в известных работах модели в достаточной степени не учитывают социально-психологические факторы субъектов и, прежде всего, их психологические состояния в процессе распространения ИПВ в социальных сетях. Распространение ИПВ происходит на основе вероятностных характеристик субъектов социальной сети и связей между субъектами. В связи с этим представляет интерес рассмотреть распространение ИПВ с учетом социально-психологических факторов субъектов социальной сети и их психологических состояний.

Целью данной работы является разработка модели процесса распространения ИПВ в социальных сетях с учетом социально-психологических факторов субъектов социальной сети и их психологических состояний на основе модели клеточного автомата. Именно клеточные автоматы, на наш взгляд, позволяют наиболее адекватно связать процесс распространения ИПВ в социальной сети и процесс изменения мнений субъектов социальной сети под влиянием их окружения с учетом социально-психологических факторов.

\section{2. МЕТОДЫ И МАТЕРИАЛЫ}

При моделировании и анализе ИПВ далее используются подход, основанный на представлении социальной сети как двумерного клеточного автомата. Двумерный клеточный автомат можно определить как множество конечных автоматов (субъектов социальной сети), размещаемых на плоскости и помечен- 
ных целочисленными координатами $(i, j)$, каждый из которых может иметь определенные свойства и находится в одном из состояний $S_{i, j} \in\left\{S_{1}, S_{2}, . ., S_{k}\right\}$. Состояние конечного автомата $(i, j)$ в момент времени $t+1$ определяется следующим образом:

$$
S_{i, j}(t+1)=F\left(S_{i, j}(t), N(i, j), t\right),
$$

где $F$ - правило изменения состояний автомата; $N(i, j)$ - окрестность точки $(i, j) ; t$ - шаг на оси времени.

В модели клеточного автомата каждая клетка изменяет свое состояние в ходе взаимодействия с ограниченным числом клеток, как правило, с теми, с которыми имеет общую сторону или вершину. Подобные модели позволяют одновременно изменять состояния всех клеток на основе общего правила клеточного автомата. Это позволяет связывать процессы, происходящие на микроуровне, с процессами пространственного взаимодействия между элементами.

В силу простоты реализации и, одновременно, возможностей моделирования сложных процессов клеточные автоматы широко применяются при моделировании систем, состоящих из большого числа частиц, которые взаимодействуют друг с другом нелинейно (динамика жидкости и газа в различных средах, пожаров, дорожного движения и т. д.), а также при описании коллективных явлений (турбулентности, упорядочения, хаоса и т. д.).

\section{1. Предлагаемая модель ИПВ в социальных сетях}

Для описания процесса распространения информационно-психологического воздействия в социальной сети предлагается следующая модель. Информационное взаимодействие в социальной сети представляется в виде двумерного клеточного автомата, решетка которого реализуется двумерным массивом, и каждая клетка нумеруется упорядоченной парой чисел $(i, j)$. Каждая клетка является субъектом социальной сети. Ближайшими соседями каждой клетки считаются клетки, имеющие с исходной общую вершину (окрестность Мура), таких клеток будет 8. Для устра- нения краевых эффектов сетка клеточного автомата топологически "сворачивается в тор" $[5,38,39]$, т. е. первая строка считается продолжением последней, а последняя - предшествующей первой. Аналогично поступают и со столбцами $[5,38,39,40,41]$.

Каждая клетка может находиться в одном из состояний: сильно выраженное позитивное отношение; нейтральное состояние (слабо выраженное негативное или позитивное отношение); сильно выраженное негативное отношение. В зависимости от состояния и социально-психологических особенностей, клетка может, как распространять информацию (оказывать влияние на соседние клетки), так и не распространять информацию $[5,38$, 39]. Изменение состояний и поведения клетки осуществляется на основе определенных в предлагаемой модели правил. Данные правила учитывают социально-психологические факторы субъектов социальной сети и их психологические состояния.

Граф-схема переходов субъекта из состояния в состояние представлена на рис. 1, где: $S_{0}$ - начальное состояние; $S_{1}$ - состояние не распространения информации $I$ совместно со своим негативным мнением (отрицательной оценкой); $S_{2}$ - состояние не распространения информации $I$ совместно со своим позитивным мнением (положительной оценкой); $S_{3}$ - состояние распространения информации $I$ совместно со своим негативным мнением (отрицательной оценкой); $S_{4}$ - состояние распространения информации $I$ совместно со своим позитивным мнением (положительной оценкой).

Каждый субъект $P_{k}$ социальной сети проявляет интерес к определенному перечню тем $T^{k}=\left\{T_{m}^{k}\right\}$, при этом остальные темы ему не интересны. Субъект $P_{k}$ обладает следующими социально-психологическими факторами [42]:

1) Собственное начальное мнение $V_{k}$ относительно информации в ИПВ, зависящее от его индивидуальных психологических особенностей, уровня образования, нравственных принципов, среды обитания и т. д. Данная величина оценивается экспертами по шкале Харрингтона, согласно которой значе- 


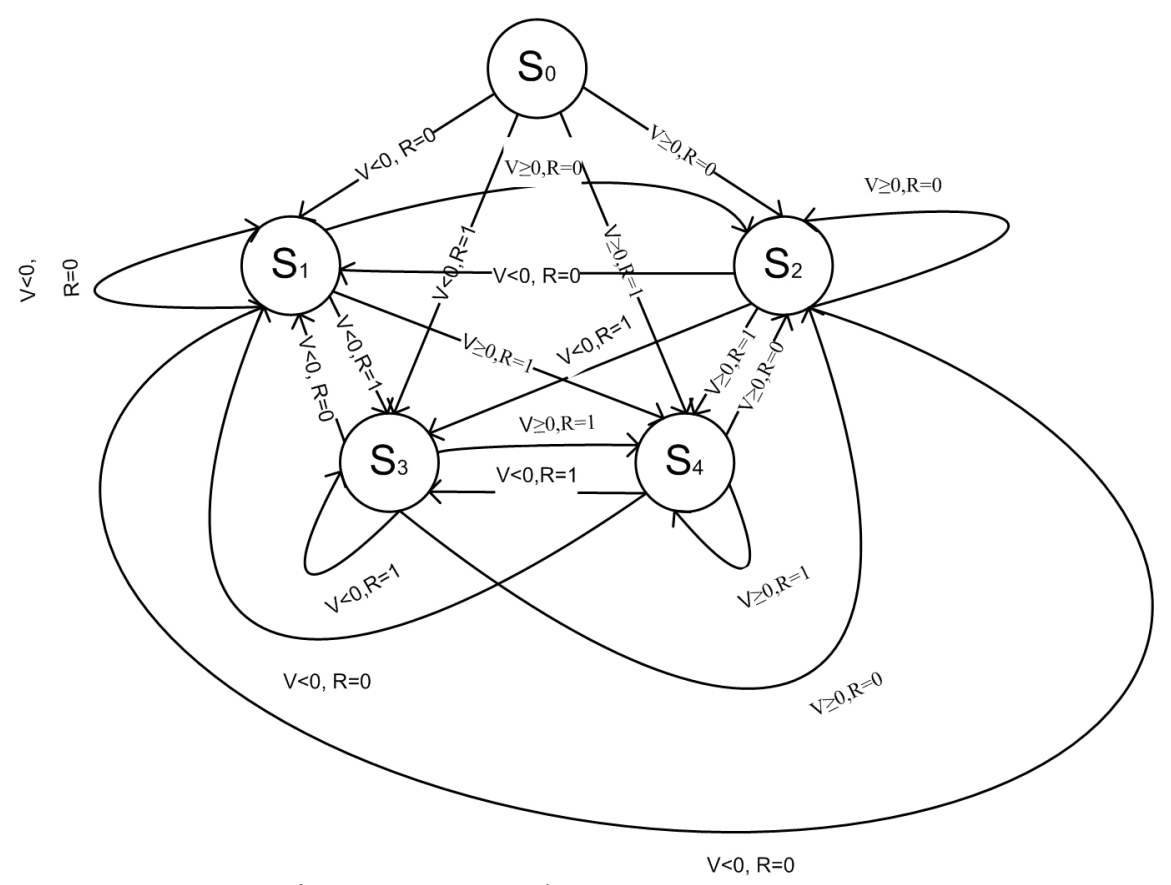

Рис. 1. Граф схема переходов из состояния в состояние

ния $V_{k}$ могут быть интерпретированы следующим образом [42]:

- диапазон $[-1 ;-0,64)$ - сильно выраженное негативное отношение, побуждающее к распространению информации $I$ совместно со своим негативным мнением (отрицательной оценкой);

- диапазон $[-0,64 ; 0)$ - слабо выраженное негативное отношение, не побуждающее к распространению информации $I$;

- диапазон $[0 ; 0,64)$ - слабо выраженное позитивное отношение, не побуждающее к распространению информации $I$;

- диапазон $[0,64 ; 1]$ - сильно выраженное позитивное отношение, побуждающее к распространению информации $I$ совместно со своим позитивным мнением (положительной оценкой).

2) Степень доверия $T R_{k j}^{T_{I}}$ к $j$-му пользователю по тематике $T$. Данная величина влияет на отношение субъекта $P_{k}$ к информации в ИПВ, полученной от $j$-го источника. Совокупность $T R_{k j}{ }^{T}$ образует «матрицу доверия» $T R^{T_{I}}$ по тематике $T_{I}$. Матрица $T R^{T_{I}}$ не обязательно должна быть симметричной.

3) Уровень коммуникабельности $O_{k}$. Данный показатель определяется на основе различных психологических тестов, например «Оценка уровня общительности» В. Ф. Ряхов- ского. Примем $O_{k}=\{$ Низкий; Средний; Высокий\} [42, 43].

4) Коэффициент передачи информации $G_{k}$, отражающий, с какой силой субъект $P_{k}$ передаст воздействие соседним субъектам.

5) Уровень восприятия информации $C_{k}$, отражающий, насколько субъект $P_{k}$ полагается на собственное мнение в рамках тематики $T_{I}$.

Для оценки текущего (в дискретный момент времени $t=t+1$ ) отношения $V_{k}^{t+1}$ к информации в ИПВ, предлагается использовать следующие соотношения [42]:

$$
\begin{aligned}
& V_{k}^{t+1}=\left\{\begin{array}{l}
1, \text { при } X \geq 1, \\
X, n p u-1<X<1, \\
-1, n p u X \leq-1,
\end{array}\right. \\
& X=V_{k}^{t}+C_{k}^{T_{I}} \cdot C S P_{k}^{t+1} \\
& C S P_{k}^{t+1}=\frac{\sum_{i=1}^{N} F_{i} T R_{k i}^{T I}}{N}, F_{i}=G_{i} V_{i}^{t},
\end{aligned}
$$

где $\operatorname{CSP}_{k}^{t+1}$ - «социальная интегральная сила», отражающая вклад в изменение мнения субъекта $P_{k}$ к информации ИПВ, полученной от субъектов, с которыми $P_{k}$ осуществляет взаимодействие; $N$ - число субъектов, взаимодействующих с субъектом $P_{k} ; F_{i}$ - сила ИПВ, с которой $i$-й субъект воздействует на субъект $P_{k}, V_{i}^{t}$ - отношение $i$-го субъекта. 
Вероятность того, что субъект $P_{k}$ будет распространять ИПВ с силой $F$, зависит от его мнения $V_{k}$ и от его уровня коммуникабельности $O_{k}$. Для определения значения передачи информации субъектом $P_{k}$ в дискретный момент времени $(t+1)$ будем использовать следующую формулу [42]:

$$
R_{k}^{t+1}=\left\{\begin{array}{r}
0, \text { если } O_{k}=\text { "Низкий" и } \\
V_{k} \in[-0,64 ; 0,64) ; \\
1, \text { иначе. }
\end{array}\right.
$$

Субъект, на которого оказано ИПВ в социальной сети, вырабатывает свое отношение к полученной информации на основе индивидуальных свойств субъекта и силы ИПВ. Данное отношение может быть как позитивным, так и негативным, и может со временем меняться под влиянием других субъектов. В зависимости от отношения субъекта к полученной информации и его уровня коммуникабельности, субъект распространяет или не распространяет полученное ИПВ.

\section{2. Алгоритм моделирования}

На рис. 2 приведена блок схема алгоритма моделирования распространения ИПВ. На подготовительном этапе определяются основные характеристики субъектов социальной сети. Результатом является построение матрицы доверия, определение уровня коммуникабельности субъектов, уровня восприятия информации, коэффициента передачи информации, сформированное начальное мнение по данному вопросу.

На первом этапе моделирования, соответствующему нулевому шагу по времени $(t=0)$, все поле состоит из клеток в состоянии $S_{0}$, за исключением нескольких клеток, которые являются инициаторами распространения ИПВ с заведомо позитивным отношением к данной информации.

На втором этапе происходит распространение информации и обмен мнениями между субъектами на шаге времени $t=t+1$, рассчитываются значения передачи информации в соответствии с формулой (2), осуществляется расчет мнений по формуле (1), клетки, чье значение передачи информации равно 1, передают информацию соседним клеткам.

Клетка может изменять свое состояние при получении воздействия $F_{i}$ от соседних клеток, для которых значение передачи информации равно 1. При получении воздействия рассчитываются текущие значения для мнения $V_{k}$ и передачи информации $R_{k}$.

\section{3. РЕЗУЛЬТАТЫ ЭКСПЕРИМЕНТАЛЬНЫХ ИССЛЕДОВАНИЙ И ИХ ОБСУЖДЕНИЕ}

Представленный алгоритм реализован на сетке размером 100 на 100. Тестирование автомата осуществлялось по следующей схеме: начальные значения распределялись по нормальному закону распределения, выбирались 10 случайных инициаторов ИПВ и 2 противника среди всех субъектов, было проведено 100 запусков автомата, в каждом запуске 1000 тактов, затем были взяты средние значения по количеству субъектов в каждом из состояний. Собственное начальное мнение субъекта $V_{k}$ относительно информации в ИПВ распределялось по нормальному закону в диапазонах $[-1 ;-0.5],[-0.5 ; 0.5],[0.5 ; 1]$. Степень доверия $T R_{k j}{ }^{T_{I}}$ распределялась по нормальному закону в диапазоне $[0 ; 1]$ или $[-1 ; 1]$. График работы автомата приведен на рис. 3-5.

На рис. 3 представлена работа автомата для случая $V_{k} \in[-0,5 ; 0,5]$, т. е. большинство субъектов имеют нейтральное отношение к ИПВ. На рис. 4 представлена работа автомата для случая $V_{k} \in[-1 ;-0,5]$, т. е. большинство субъектов имеют негативное отношение к ИПВ. На рис. 5 представлена работа автомата для случая $V_{k} \in[0,5 ; 1]$, т. е. большинство субъектов имеют позитивное отношение к ИПВ. На рисунках «а» представлена работа автомата для случая $T R_{k j}{ }^{T_{I}} \in[0 ; 1]$, т. е. субъекты принимают мнения взаимодействующих с ними субъектов. На рисунках «б» представлена работа автомата, для случая $T R_{k j}{ }^{T_{I}} \in[-1 ; 1]$, т. е. субъект может принимать противоположное мнение от навязываемого в процессе ИПВ.

Анализ рис. 3-5 показывает, что:

- процесс распространения ИПВ в социальной сети носит характер, близкий к экспоненциальному; 


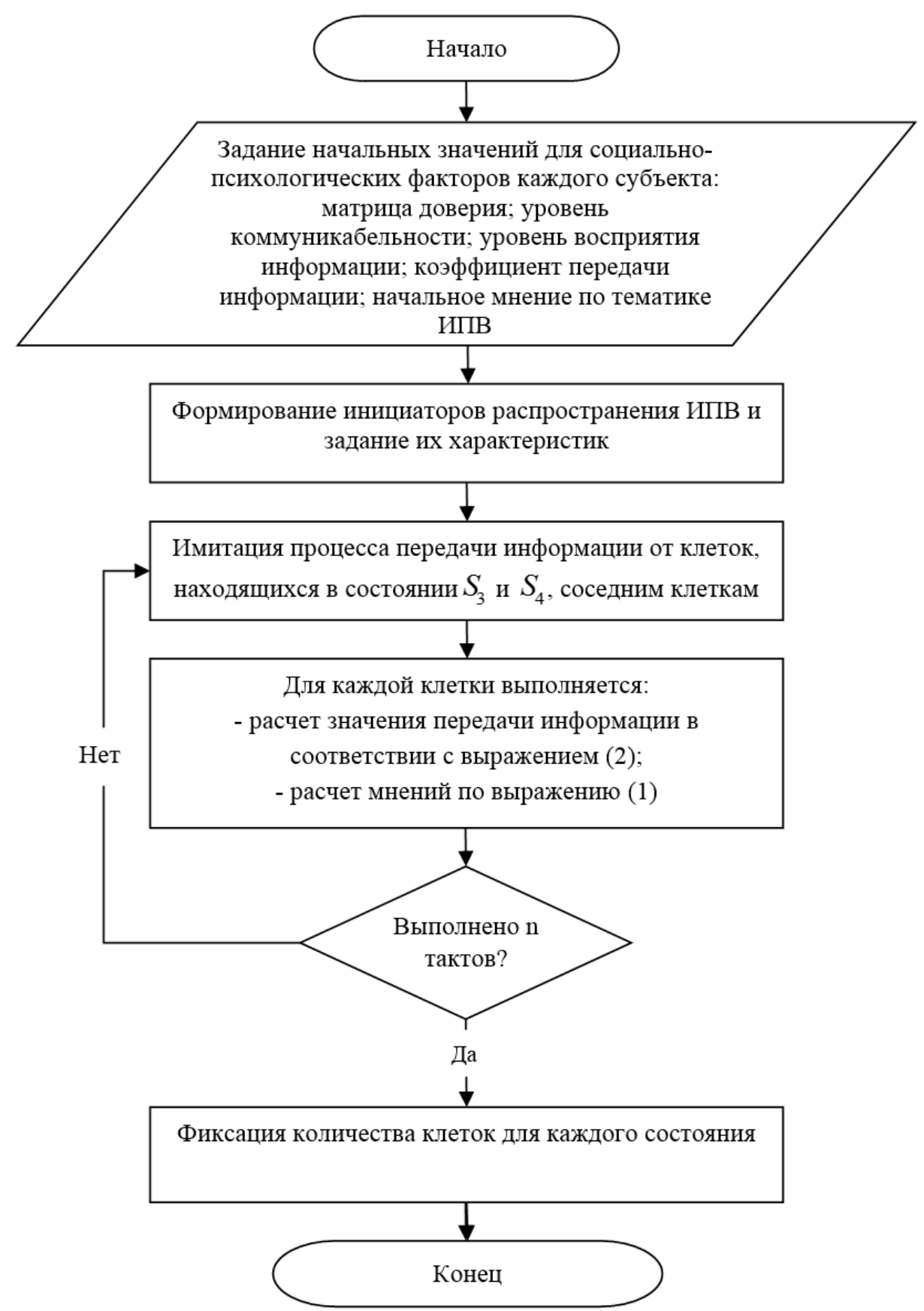

Рис. 2. Обобщенная блок схема алгоритма моделирования распространения ИПВ

- в случае нейтрального отношения (рис. 3а) субъектов к информации, распространяемой в ходе ИПВ, небольшого числа инициаторов достаточно для успешной реализации ИПВ;

- в случае заведомо негативного или позитивного отношения (рис. 4а и 5а) субъектов к информации, распространяемой в ходе ИПВ, их мнение относительно информации не изменяется;

- в случае, когда субъекты могут не доверять друг другу и изменять свое мнение на противоположное (рис. 36, 46 и 56), то количество субъектов в состояния S3 и S4 совпадает, независимо от их начального состояния.

Результаты, полученные с помощью предложенной модели, совпадают с результатами работ $[4,5,30,38]$ по распространению информации, что является частным случаем распространения ИПВ в социальных сетях. В отличие от работ $[4,5,30,38,40,41]$, данная модель учитывает социально-психологические факторы субъектов и их психологиче- 
Моделирование прочессов информационно-психологического воздействия в соииальных сетях

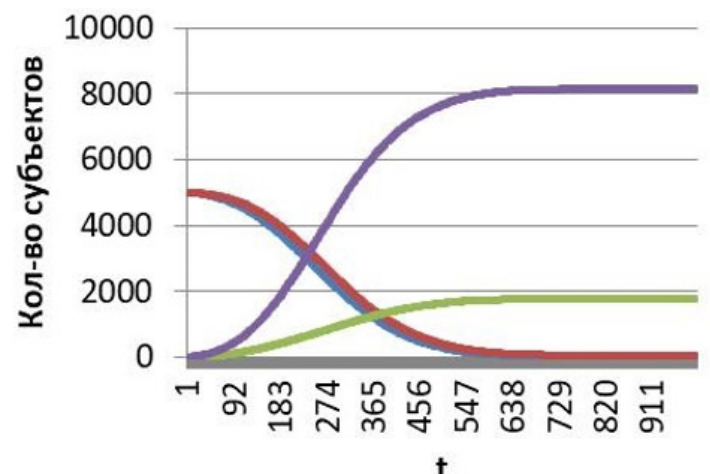

$a$

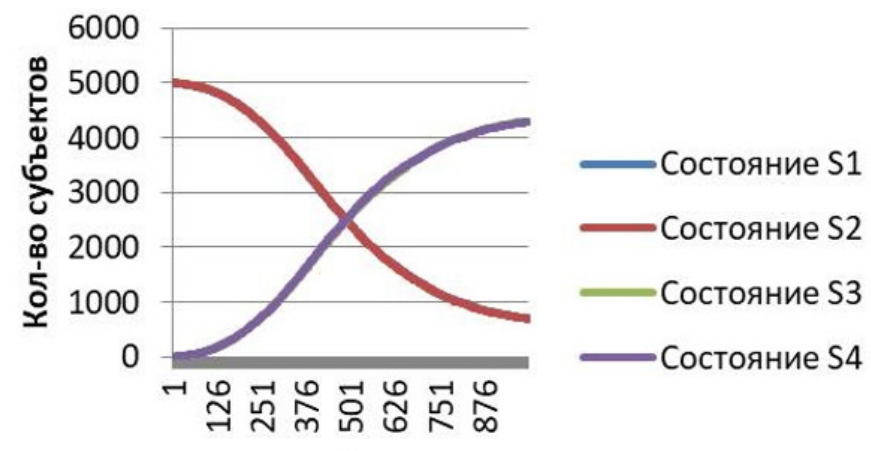

6

Рис. 3. Распределение клеток в зависимости от дискретного времени, для $V_{k} \in[-0,5 ; 0,5]$
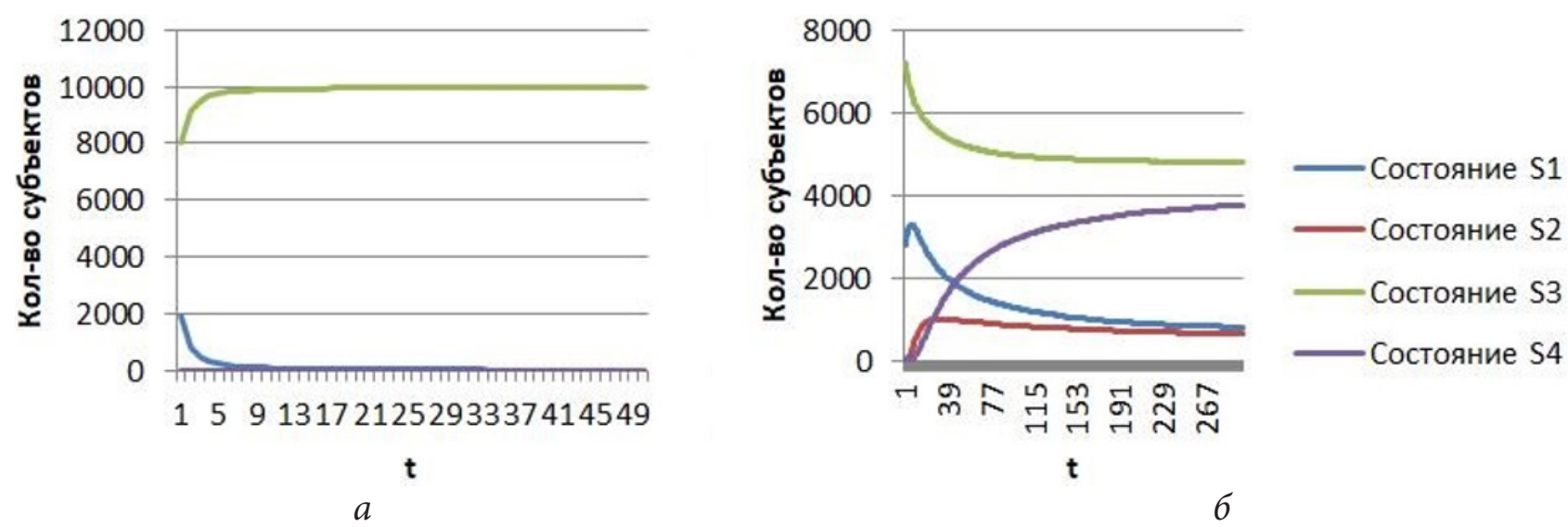

Рис. 4. Распределение клеток в зависимости от дискретного времени, для $V_{k} \in[-1 ;-0,5]$
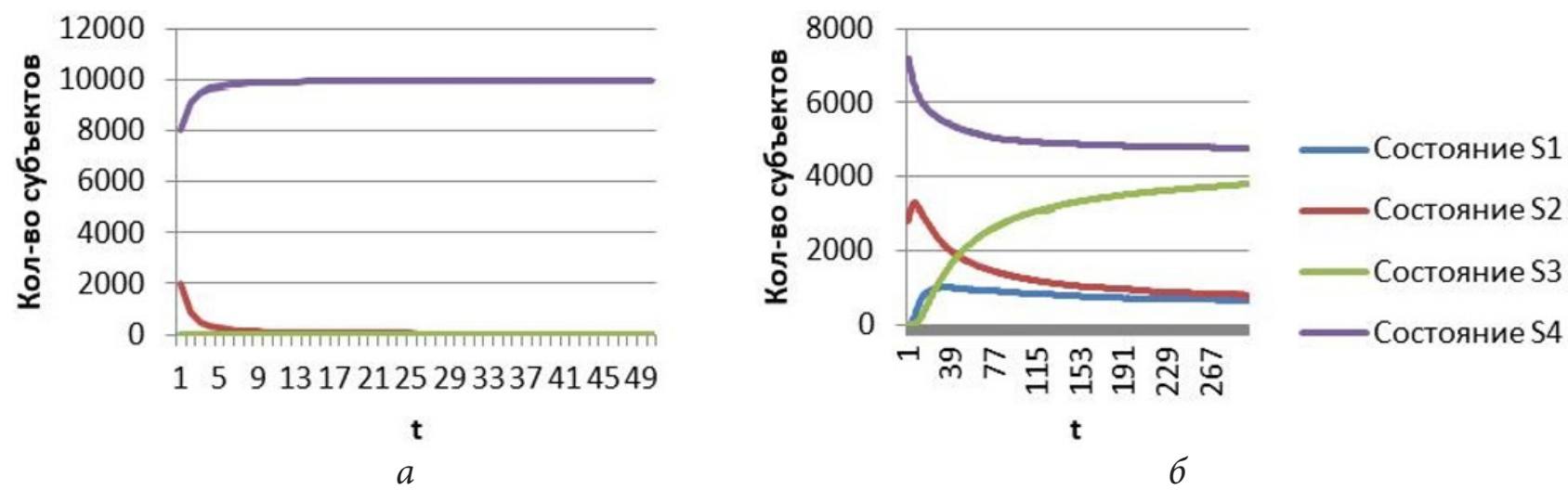

Рис. 5. Распределение клеток в зависимости от дискретного времени, для $V_{k} \in[0,5 ; 1]$

ские состояния в процессе распространения ИПВ в социальных сетях, а не вероятностные характеристики субъектов социальной сети.

\section{ЗАКЛЮЧЕНИЕ}

В данной работе предложена модель распространения информационно-психологического воздействия с использованием клеточных автоматов. Модели клеточных автоматов позволяют изменить состояние большого числа клеток за предельно короткое время, что можно использовать при моделировании процесса распространения информационно-психологического воздействия в социальных сетях. Предложенная модель позволяет связать процесс распространения ИПВ в социальной сети и процесс изменения состояний субъектов социальной сети под влиянием их окружения с учетом социально-психологических факторов. 


\section{СПИСОК ЛИТЕРАТУРЫ}

1. Баришполеи, В. А. Области применения информационно-психологического воздействия / В. А. Баришполец // Информационные технологии. - 2014. - № 1. - С. 52-79.

2. Баришполеи, В. А. Информационно-психологическая безопасность: основные положения / В. А. Баришполец // Информационные технологии. - 2013. - № 2. - С. 62-104.

3. Доктрина информационной безопасности Российской Федерации, утверждена Указом Президента Российской Федерации от 5 декабря 2016 г. № 646. - Режим доступа: http://static.kremlin.ru/media/acts/ files/0001201612060002.pdf (Дата обращения 22.01.2018).

4. Губанов, Д. А. Модели влияния в социальных сетях / Д. А. Губанов, Д. А. Новиков, А. Г. Чхартишвили // Управление в социально-экономических системах. - 2008. - № 27. C. 205-281.

5. Додонов, А. Г. Моделирование информационных резерваций / А. Г. Додонов, Д. В. Ландэ // Открытые семантические технологии проектирования интеллектуальных систем. - 2016. - № 1. - С. 253-256.

6. Bailey, N. The Mathematical Theory of Infectious Diseases and Its Applications / N. Bailey. - New York : Hafner Press, 1975. - 429 p.

7. Golub, B. Naive Learning in Social Networks: Convergence, Influence and the Wisdom of Crowds / B. Golub, M. Jackson. // American Economic Journal: Microeconomics. - 2010. № 2:1. - P. 112-149.

8. Granovetter, M. The Strength of Weak Ties / M. Granovetter // American Journal of Psychology. - 1973. - №78(6). - P. 1360-1380.

9. Evendar, E. A Note on Maximizing the Spread of Influence in Social Networks / E. Evendar., A. Shapira // Internet and Network Economics. - 2007. - P. 281-286.

10. Kempe, D. Maximizing the Spread of Influence through a Social Network / D. Kempe, J. Kleinberg, E. Tardos // Proceedings of the 9-th ACM SIGKDD International Conference on Knowledge Discovery and Data Mining. 2003. - P. 137-146.
11. Masuda, N. Analysis of relative influence of nodes in directed networks / N. Masuda, Y. Kawamura, H. Kori // Physical Review. - 2009. E 80. - P. 46-114.

12. Rolfe, M. Social Networks and Threshold Models of Collective Behavior / M. Rolfe. Preprint. - Chicago: University of Chicago. - 2004. P. 1420 - 1443.

13. Valente, T. Network Models of the Diffusion of Innovations / T. Valente. - Cresskill, NJ: Hampton Press. - 1995. - P. 480-488.

14. Гончаров, И. В. Формализация процесca информационно-психологического воздействия / И. В. Гончаров, Н. Ю. Демьяненко, Я. С. Мишина // Вестник Воронеж. гос. ун-та. Сер. Системный анализ и информационные технологии. - 2012. № 2. - С. 36-41.

15. Гончаров, И. В. Возможность моделирования процесса информационно-психологического воздействия с помощью нейронных сетей / И. В. Гончаров, Н. Ю. Демьяненко, Я. С. Мишина // XIII Международная научно-методическая конференция «Информатика: проблемы, методология, технологии». Воронеж, 2013. - С. 124-130.

16. Гончаров, И. В. Анализ возможностей и систематизация технических средств, характеризующих построение канала информационно-психологического воздействия / И. В. Гончаров, Н. Ю. Демьяненко, А. О. Хачумов, С. С. Ноздрачев // Труды Российской научно-технической конференции. - Воронеж, 2009. - C. $168-174$.

17. Гончаров, И. В. Описание подхода к представлению состояний объектов и субъектов процесса информационно-психологического воздействия с помощью вейвлет-преобразования / И. В. Гончаров, Я. С. Мишина // Международная научно-практическая конференция «Техника и безопасность объектов уголовно-исполнительной системы - 2013». ФКОУ ВПО Воронежский институт ФСИН России. - 2013. - С. 58-70.

18. Friedkin, N. E. Structural Cohesion and Equivalence Explanations of Social Homogeneity / N. E. Friedkin // Sociological Methods and Research. - 1984. - № 12. - P. 235-261. 
19. Leenders, $R$. The Specification of Weight Structures in Network Autocorrelation Models of Social Influence / R. Leenders. - 2002. - p. 46.

20. Бреер, В. В. Стохастические модели социальных сетей / В. В. Бреер // Управление большими системами. - 2009. - № 27. C. 169-204.

21. Tarnow, E. Like Water and Vapor - Conformity and Independence in the Large Group / E. Tarnow. - 1996. - P. 136-151.

22. Berger, R. L. A Necessary and Sufficient Conditions for Reaching a Consensus using De Groot's method / R. L. Berger // Journal of American Statistical Assotiation. - 1981. - V. 76. P. 415-419.

23. Chatterjee, S. Toward Consensus: Some Convergence Theorems on Repeated Averaging / S. Chatterjee, E. Seneta // Journal of Applied Probability. - 1977. - № 14. - P. 159-164.

24. De Marzo, P. Persuasion Bias, Social Influence and Unidimensional Opinions / P. De Marzo, D. Vayanos, J. Zwiebel // Quarterly Journal of Economics. - 2003. - № 118(3). - P. 909-968.

25. Hesgelman, R. Opinion Dynamics and Bounded Confidence Models, Analysis and Simulation / R. Hesgelman, U. Krause // Journal of Artificial Societies and Social Simulation. 2002. - № 5:3 - P. 44-77.

26. Казаков, В. Г. Многоагентные системы и сценарии развития Веб / В. Г. Казаков, И. Н. Карпенко // Информационное общество: образование, наука, культура и технологии будущего. - 2017. -№ 1. - С. 34-43.

27. Охапкина, Е. П. Разработка фрагмента онтологии для многоагентной системы модерации сообщений пользователей / Е. П. Охапкина, Л. И. Воронова // Вестник НГУ, Серия: Информационные технологии. - 2014. № 1. - С. 54-62.

28. Казаков, В. Г. Социальные сети и многоагентные системы / В. Г. Казаков, И. Н. Карпенко // Информационное общество: образование, наука, культура и технологии будущего. - 2015. - №1. - С. 260-268.

29. Domingos, $P$. Mining the Network Value of Customers / P. Domingos, M. Richardson // Proceedings of the Seventh International Conference on Knowledge Discovery and Data Mining. -2002 . - P. 57-66.
30. Ландэ, Д. В. Модель диффузии информации / Д. В. Ландэ // Информационные технологии и безопасность. Менеджмент информационной безопасности. Сборник научных трудов Института проблем регистрации информации. - 2007. -№ 10 - С. 51-67.

31. Зайцев, И. Д. Многоагентные системы в моделировании социально-экономических отношений: исследование поведения и верификации свойств с помощью цепей Маркова / И. Д. Зайцев // Материалы LI международной научной студенческой конференции «Студент и научно-технический прогресс», Информационные технологии. - Новосибирск. - 2013. - С. 143.

32. Goldenberg, J. Talk of the Network: A Complex Systems Look at the Underlying Process of Word-of-Mouth / J. Goldenberg, B. Libai, E. Muller // Marketing Letters. - 2001. - № 2. P. 11-34.

33. Romualdo, P. Epidemic Spreading in ScaleFree Networks / P. Romualdo., V. Alessandro // Physical Review Letters. - 2001. - № 14(86). P. 3200-3203.

34. Wu, F. Information Flow in Social Groups / F. Wu, B. Huberman, L. Adamic, J. Tyler // Statistical and Theoretical Physics. - 2004. - № 337. P. 327-335.

35. Рунков, Я. К. Использование нейронных сетей в социальных коммуникациях и интернет-ресурсах, их обеспечивающих / Я. К. Рунков // Международный журнал экспериментального образования. - 2015. № 12. - С. 626-627.

36. Нейронные сети и социальная инженерия, Идеи и перспективы. - Режим доступа: http://ai-news.ru/2017/07/nejronnye_seti_i_ socialnaya_inzheneriya_idei_i_perspektivy.html (Дата обращения: 22.01.2018).

37. Leskovec, J. Cost-effective Outbreak Detection in Networks / J. Leskovec, A. Krause, C. Guestrin, C. Faloutsos, J. Vanbriesen, N. Glance // Proceedings of the 13-th ACM SIGKDD International Conference on Knowledge Discovery and Data Mining. - 2007. - P. 420-429.

38. Ландэ, Д. В. Модель диффузии информации // Информационные технологии и безопасность. Менеджмент информационной безопасности. Сборник научных трудов 
Института проблем регистрации информации. - Вып. 10. - 2007. - С. 51-67.

39. Шмидт, Ю. Д. О некоторых подходах к моделированию пространственной диффузии инноваций / Ю. Д. Шмидт, О. Н. Лободина // Пространственная Экономика. - 2015. № 2. - С. 103-115.

40. Ломакин, С. Г. Анализ модели передачи информации в сети клеточных автоматов / С. Г. Ломакин, А. М. Федотов // Вестник НГУ, Серия: Информационные технологии. 2014. - № 3. - С. 86-99.

41. Нижегородиев, Р. М. Модели клеточных автоматов в теории диффузии инноваций /

Гончаров И. В. - канд. техн. наук, доцент, Воронежский государственный университет. E-mail: goncharov@infobez.org

Паринов П. А. - аспирант кафедры технологий обработки и защиты информации, факультет компьютерных наук, Воронежский государственный университет.

E-mail: parinov_p@sc.vsu.ru

Сирота А. А. - д-р техн. наук, профессор, заведующий кафедрой технологий обработки и защиты информации, факультет компьютерных наук, Воронежский государственный университет.

E-mail: sir@cs.vsu.ru
Р. М. Нижегородцев, В. Д. Секерин, С. В. Лисафьев,// Вопросы новой экономики. - 2012. № 3. - С. 39-43.

42. Ажмухамедов, И. М. Моделирование процесса распространения информации в социальных сетях / И. М. Ажмухамедов, Д. А. Мачуева, Д. А. Жолобов // Фундаментальные исследования. - 2017. - № 5. - С. 9-14.

43. Гребень, Н. Ф. Психологические тесты для профессионалов / Н. Ф. Гребень - Минск : Современная школа, 2007. - 496 с.

Goncharov I. V. - Ph.D., Associate Professor, Voronezh State University.

E-mail: goncharov@infobez.org

Parinov P. A. - post-graduate student of the Department of Technology of Information Processing and Protection, Faculty of Computer Science, Voronezh State University.

E-mail: parinov_p@sc.vsu.ru

Sirota A. A. - Doctor of Technical Sciencies, Professor, Department of Processing Technology and Information Security, Computer Sciences Faculty, Voronezh State University.

E-mail: sir@cs.vsu.ru 